\title{
Relato de experiência de trabalho com saúde bucal indígena no Vale do Javari, Amazonas, Brasil
}

\author{
Report of work experience with indigenous oral health in the Javari Valley, Amazonas, Brazil \\ Informe de experiencia laboral con salud bucal indígena en el Valle de Javari, Amazonas, Brasil \\ Liliane Cristina BARBOSA ${ }^{1}$ \\ Tânia Adas SALIBA ${ }^{2}$
}

${ }^{1}$ Mestranda, Programa de Pós-Graduação em Odontologia Preventiva e Social, Universidade Estadual Paulista (UNESP), Faculdade de Odontologia de Araçatuba, 16015-050 Araçatuba-SP, Brasil

${ }^{2}$ Professora Associada, Coordenadora do Programa de Pós-Graduação em Odontologia Preventiva e Social, Universidade Estadual Paulista (UNESP), Faculdade de Odontologia de Araçatuba, 16015-050 Araçatuba-SP, Brasil

\section{Resumo}

O Vale do Javari é uma reserva indígena, localizada no município de Atalaia do Norte no oeste do Amazonas, no Brasil com 3.700 indígenas das etnias Marubo, Mayuruna, Matis, Kanamary, Kulina e Korubo. O presente trabalho objetivou-se a descrever a organização dos serviços de saúde bucal indígena, a programação em saúde bucal e os resultados obtidos pelas equipes de atuação nas aldeias, no período de 3 anos. A pesquisa do tipo qualitativa, descritiva tratou-se de análise documental de relatórios das equipes do Distrito Sanitário Especial Indígena (DSEI) Vale do Javari. De acordo com as diretrizes das ações para atenção à saúde bucal nos DSEIs, os serviços são organizados em três fases: Controle de infecção intrabucal ( $1^{\mathrm{a}}$ fase), Reabilitação ( $2^{\mathrm{a}}$ fase) e Assistência especializada ( $3^{\mathrm{a}}$ fase). Aproximadamente $50 \%$ da população recebeu atendimento odontológico neste período e em $97,93 \%$, dessa população atendida, a $1^{\circ}$ fase de tratamento foi concluída. Dentre os procedimentos mais frequentes, destacou-se: restaurações atraumáticas, restaurações convencionais, extrações e ações coletivas compostas por reuniões com a comunidade, palestras de promoção e prevenção de saúde bucal, escovação supervisionada e visitas domiciliares. Diante de uma população com necessidades complexas e dificuldades logísticas, este estudo contribuiu com uma visão sobre a experiência dos cirurgiões dentistas, que deve ser considerada parte interessada e importante na implementação e sustentabilidade do programa de saúde bucal para os povos indígena.

Descritores: População Indígena; Serviços de Saúde do Indígena; Saúde Bucal.

\begin{abstract}
The Javari Valley is an indigenous reserve, located in Atalaia do Norte in western Amazonas, Brazil with 3,700 Marubo, Mayuruna, Matis, Kanamary, Kulina and Korubo ethnic groups. The present study aimed to describe the organization of indigenous oral health services, oral health programming and the results obtained by the village teams in the 3-year period. The qualitative, descriptive research was a documentary analysis of the reports of the teams of the Indigenous Special Sanitary District (DSEI) Vale do Javari. According to the oral health care guidelines in the DSEIs, the services are organized in three phases: Intra-oral infection control (1st phase), Rehabilitation (2nd phase) and Specialized care (3rd phase). Approximately 50\% of the population received dental care in this period and in $97.93 \%$ of this population served, the first phase of treatment was completed. Among the most frequent procedures were: atraumatic restorations, conventional restorations, extractions and collective actions composed of meetings with the community, oral health promotion and prevention lectures, supervised brushing and home visits. Faced with a population with complex needs and logistical difficulties, this study contributed with an insight into the experience of dentists, who should be considered an interested and important stakeholder in the implementation and sustainability of the oral health program for indigenous peoples.
\end{abstract}

Descriptors: Indigenous Population (Public Health); Health Service, Indigenous; Oral Health.

\section{Resumen}

El Valle Javari es una reserva indígena ubicada en el municipio de Atalaia do Norte, en el oeste de Amazonas, Brasil, con 3,700 indígenas Marubo, Mayuruna, Matis, Kanamary, Kulina y Korubo. Este documento tuvo como objetivo describir la organización de los servicios de salud bucal indígenas, la programación de salud bucal y los resultados obtenidos por los equipos que trabajan en las aldeas durante un período de 3 años. La investigación cualitativa y descriptiva fue un análisis documental de los informes de los equipos del Distrito Sanitario Indígena Especial Vale do Javari (DSEI). De acuerdo con los lineamientos de acciones para la atención de la salud bucal en las DSEI, los servicios se organizan en tres fases: Control de Infecciones Intraabucales ( $1^{\mathrm{a}}$ fase), Rehabilitación $\left(2^{\mathrm{a}}\right.$ fase $)$ y Atención Especializada $\left(3^{\mathrm{a}}\right.$ fase). Aproximadamente el 50\% de la población recibió atención dental durante este período y en el 97,93\% de esta población atendida, se completó la primera fase de tratamiento. Entre los procedimientos más frecuentes se encontraban: restauraciones atraumáticas, restauraciones convencionales, extracciones y acciones colectivas compuestas por reuniones con la comunidad, conferencias sobre prevención y prevención de la salud oral, cepillado supervisado y visitas domiciliarias. Dada una población con necesidades complejas y dificultades logísticas, este estudio contribuyó con una visión de la experiencia de los cirujanos dentales, que debe considerarse una parte importante e interesada en la implementación y sostenibilidad del programa de salud oral para los pueblos indígenas.

Descriptores: Población Indígena; Servicios de Salud del Indígena; Salud Bucal.

\section{INTRODUÇÃO}

O Vale do Javari é uma reserva indígena, localizada no município de Atalaia do Norte no oeste do Amazonas, Brasil, com extensão territorial de 8.457.000 hectares, 3.700 indígenas espalhados por 50 aldeias, nas quais habitam os povos de etnia Marubo, Mayuruna, Matis, Kanamary, Kulina e Korubo.

Estima-se que atualmente vivam no Brasil pelo menos 246 povos indígenas, falantes de 150 línguas ${ }^{7}$. Segundo dados do IBGE $^{2}$, cerca de $78 \%$ dessa população está localizada nos estados das regiões Norte $(37 \%)$, Nordeste $(25 \%)$ e Centro-Oeste
(16\%), que também c ontém a maior parte das terras indígenas que hoje estão em alguma fase do processo administrativo de demarcação $(57 \%, 12 \%$ e $21 \%$ respectivamente) ${ }^{3}$.

Apesar do Sistema Único de Saúde (SUS) ter sido criado em 1988, e sua Lei Orgânica datar de 1990, o Subsistema de Atenção à Saúde Indígena (SASI) só foi criado em 1999, por meio da Lei 9.836/1999, do deputado federal Sérgio Arouca, porém, se a luta indígena pela saúde se fortaleceu nesse contexto, a literatura especializada mostra que há fragilidades tanto na efetivação dos seus direitos 
sociais e territoriais quanto do direito à saúde ${ }^{11}$.

Segundo o Censo $2010^{8}$, atualmente cerca de 817 mil pessoas se autodeclaram indígenas no Brasil. Enquanto a população brasileira cresceu cerca de $30 \%$ nos últimos 20 anos, a parcela indígena cresceu $177 \%$. Esses dados apontam para uma tendência de reversão do decrescimento demográfico que no passado ameaçava os povos indígenas no Brasil ${ }^{1}$.

De acordo com a Política Nacional de Atenção à Saúde dos Povos Indígenas (PNASPI) ${ }^{9}$, a organização do DSEI é orientada para ser um espaço etnocultural dinâmico, geográfico, populacional e administrativo bem delimitado, responsável pela realização de atividades no território indígena, visando medidas racionalizadas e qualificadas de atenção à saúde, promovendo a reordenação da rede e das práticas sanitárias, e desenvolvendo atividades administrativo-gerenciais necessárias à prestação da assistência aos povos indígenas. A PNASPI estabelece que as equipes de saúde dos distritos devam ser compostas por médicos, enfermeiros, odontólogos, auxiliares de enfermagem e agentes indígenas de saúde.

Os DSEIs são organizados em polos-base, em aldeias estrategicamente definidas, e são estruturados para oferecer apenas atenção primária, referenciando os casos de média e alta complexidade para unidades do SUS localizadas nos perímetros urbanos ou em outros municípios.

Há poucas publicações ou estudos que retratam ou avaliam a experiência dos profissionais na saúde indígena, por este motivo, identifica-se como necessário o aprofundamento de reflexões acerca dos benefícios e dos desafios que a presença significativa da equipe de saúde bucal em área indígena representa para a situação de saúde dessas populações, com essa perspectiva o objetivo deste trabalho foi descrever a organização dos serviços de saúde bucal indígena, a programação em saúde bucal e os resultados obtidos pelas equipes de atuação nas aldeias do Distrito Sanitário Especial Indígena - Vale do Javari, Amazonas, Brasil, no período de 3 anos.

\section{MATERIAL E MÉTODO}

No DSEI Vale do Javari, com 3700 indígenas, habitam os povos de etnia Marubo, Mayuruna, Matis, Kanamary, Kulina e Korubo, com 50 aldeias agrupadas em sete polos-base denominados: Itacoaí, Médio Javari, Médio Curuçá, Médio Ituí, Alto Ituí, Alto Curuçá e Jaquirana. Os atendimentos eram realizados nas aldeias $\mathrm{e}$ posteriormente consolidados nos relatórios do polobase de referência. A pesquisa foi do tipo qualitativa, de análise documental dos relatórios das equipes, cujos dados foram analisados por meio de estatística descritiva.

\section{RESULTADOS}

\section{- Procedimentos individuais}

De acordo com as diretrizes das ações para atenção à saúde bucal nos DSEIs, os serviços estão organizados em três fases: $1^{\text {a }}$ Fase: Controle de infecção intrabucal; $2^{\mathrm{a}}$ Fase: Reabilitação e $3^{\mathrm{a}}$ Fase: Assistência especializada.

A Figura 1 mostra o atendimento clínico realizado na escola da aldeia Flores, da etnia Mayuruna, pois polo-base ainda não estava construído, o local foi adaptado para utilizar a cadeira portátil e autocláve usando gerador de energia para seu funcionamento. Na aldeia São Sebastião de etnia Marubo, o atendimento foi realizado no polo-base inacabado, também utilizando gerador de energia para o funcionamento dos equipamentos portáteis (Figura 2).

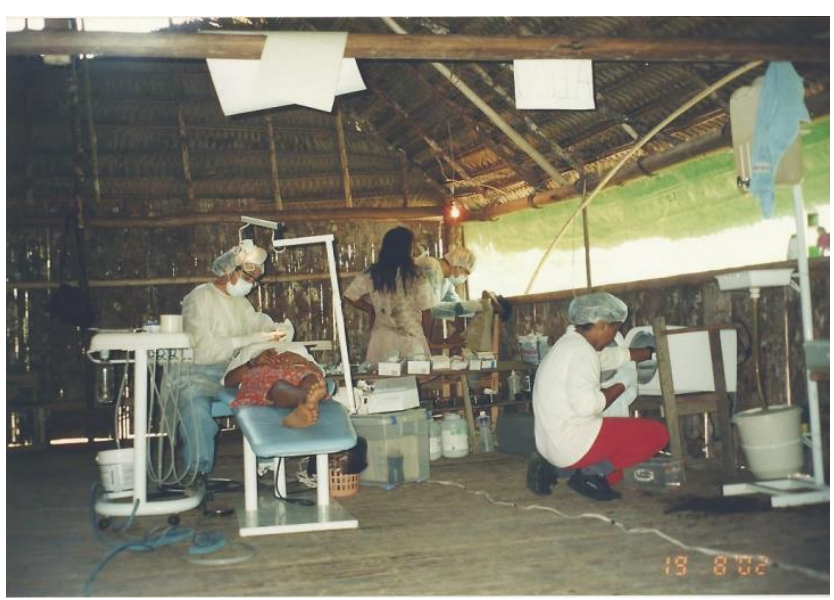

Figura1: Atendimento clínico na aldeia Flores de etnia Mayuruna do DSEI Vale do Javari-AM, Brasil.

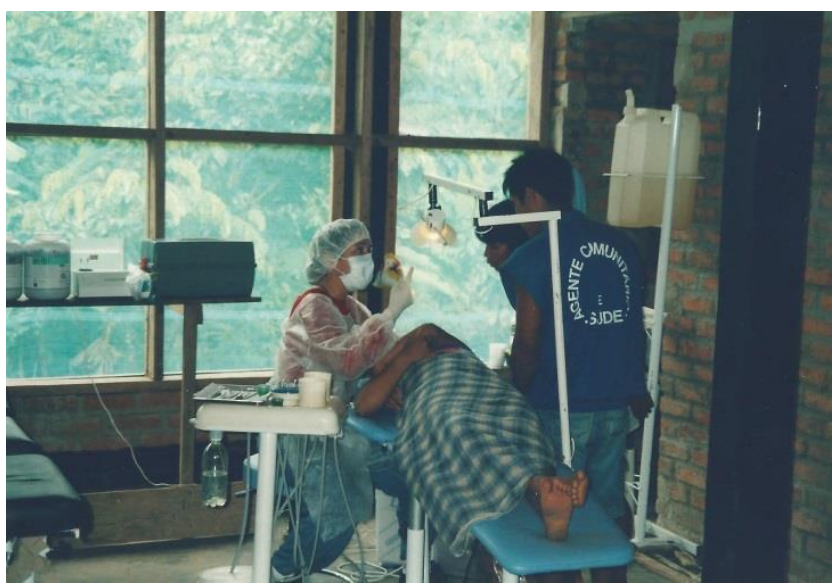

Figura 2: Atendimento clínico na aldeia São Sebastião de etnia Marubo do DSEI Vale do Javari-AM, Brasil.

Dentre os diversos procedimentos odontológicos, os profissionais realizavam, em sua maioria, restaurações pela técnica ART (Atraumatic Restorative Treatment), além das convencionais restaurações em amálgama e resina composta fotopolimerizável, exodontias, raspagens periodontais e alguns casos de selantes e pulpotomias. Para quantificar os procedimentos realizados, a Tabela 1 
mostra a distribuição em cada um dos sete polos-base de referência.

Dos 1494 indígenas com problemas bucais identificados, 1463 tiveram a primeira fase da atenção odontológica concluídas, o que resultou no controle da infecção intrabucal por meio dos procedimentos coletivos e individuais e em um índice médio de $97,93 \%$ de produtividade (Tabela 2 ).

Tabela 1. Procedimentos odontológicos realizados na população indígena em cada polo base do DSEI Vale do Javari

\begin{tabular}{lcccccccc}
\hline Procedimento & $\begin{array}{c}\text { Alto } \\
\text { Curuçá }\end{array}$ & $\begin{array}{c}\text { Médio } \\
\text { Curuçá }\end{array}$ & $\begin{array}{c}\text { Alto } \\
\text { Ituí }\end{array}$ & $\begin{array}{c}\text { Médio } \\
\text { Ituí }\end{array}$ & $\begin{array}{c}\text { Médio } \\
\text { Javari }\end{array}$ & Itacoaí & Jaquirana & Total \\
\hline Consultas & 177 & 206 & 270 & 274 & 250 & 209 & 1132 & 2518 \\
ART & 213 & 185 & 309 & 280 & 342 & 37 & 1461 & 2827 \\
Amálgama & 60 & 123 & 68 & 71 & 106 & 121 & 1003 & 1552 \\
$\begin{array}{l}\text { Resina } \\
\text { Composta }\end{array}$ & 38 & 39 & 2 & 2 & 60 & 63 & 198 & 402 \\
$\begin{array}{l}\text { Exodontia } \\
\text { Decíduo }\end{array}$ & 53 & 51 & 120 & 177 & 112 & 44 & 472 & 1029 \\
$\begin{array}{l}\text { Exodontia } \\
\text { Permanente }\end{array}$ & 89 & 136 & 242 & 156 & 156 & 520 & 1226 & 2525 \\
Raspagem & 78 & 124 & 89 & 94 & 7 & 145 & 307 & 844 \\
Outros & 0 & 0 & 4 & 1 & 1 & 0 & 22 & 28 \\
\hline Total & $\mathbf{7 0 8}$ & $\mathbf{8 6 4}$ & $\mathbf{1 1 0 4}$ & $\mathbf{1 0 5 5}$ & $\mathbf{1 0 3 4}$ & $\mathbf{1 1 3 9}$ & $\mathbf{5 8 2 1}$ & $\mathbf{1 1 7 2 5}$ \\
\hline
\end{tabular}

Fonte: Prontuários Odontológicos do DSEI Vale do Javari

Tabela 2. Produtividade por polo-base $-1^{\text {a }}$ fase de tratamento concluída

\begin{tabular}{lcccc}
\hline $\begin{array}{c}\text { Polo } \\
\text { Base }\end{array}$ & $\begin{array}{c}\text { População } \\
\text { Geral }\end{array}$ & $\begin{array}{c}\text { Pessoas com problemas } \\
\text { identificados }\end{array}$ & $\begin{array}{c}\mathbf{1}^{\mathbf{a}} \text { Fase do } \\
\text { tratamento concluída }\end{array}$ & Produtividade \\
\hline Itacoaí & 367 & 142 & 138 & $97,18 \%$ \\
$\begin{array}{l}\text { Médio } \\
\text { Javari }\end{array}$ & 361 & 134 & 131 & $97,76 \%$ \\
$\begin{array}{l}\text { Médio } \\
\text { Curuçá }\end{array}$ & 290 & 167 & 160 & $95,81 \%$ \\
Médio & & & 129 & $96,99 \%$ \\
Ituí & 355 & 133 & 242 & $98,37 \%$ \\
Alto Ituí & 456 & 246 & 115 & $98,29 \%$ \\
Alto & & & 548 & $98,74 \%$ \\
Curuçá & 266 & 117 & $\mathbf{1 4 6 3}$ & $\mathbf{9 7 , 9 3 \%}$ \\
Jaquirana & 773 & 555 & & \\
\hline \multicolumn{1}{c}{ Total } & $\mathbf{2 8 6 8}$ & $\mathbf{1 4 9 4}$ & & \\
\hline Fonte: Prontuários Odontológicos do DSEI Vale do Javari. &
\end{tabular}

\section{- Procedimentos coletivos}

Quanto às atividades preventivas, a primeira fase (controle de infecção intrabucal) preconiza a utilização de flúor gel nas escovas de dentes, podendo ser executada de duas formas: 06 aplicações com intervalos de 3 dias (72h) ou 06 aplicações semanais consecutivas. A segunda opção foi a escolhida, porém somente com as crianças de 05 a 12 anos; os trabalhos foram iniciados pela equipe de saúde bucal e nas semanas seguintes realizados pelos agentes indígenas de saúde - AIS, os quais notificavam os registros, à equipe responsável, via rádio (Gráfico 1 e Figuras 3,4 e 5).

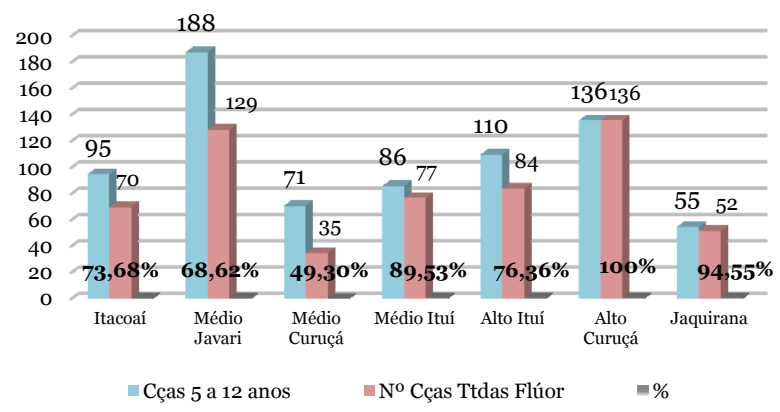

Gráfico 1: Percentual de cobertura no programa de escovação supervisionada.

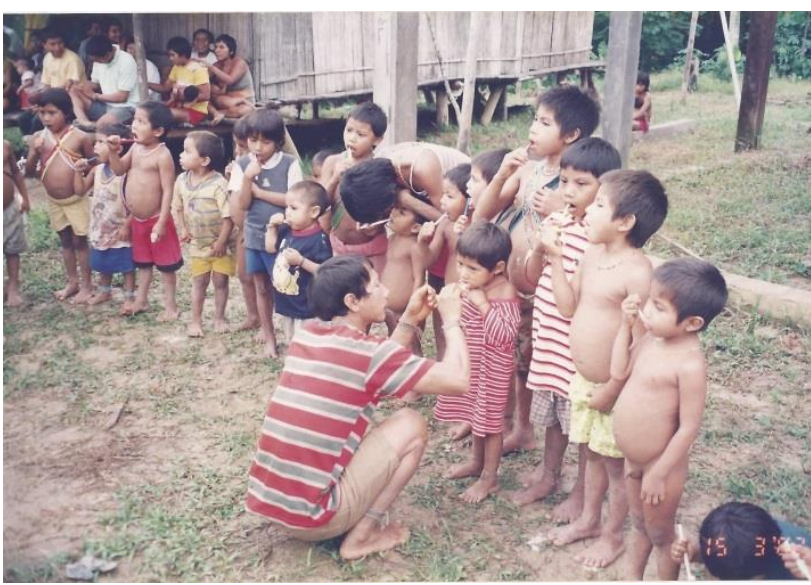

Figura 3: Capacitação do AIS, durante ação coletiva de escovação supervisionada, na aldeia Aurélio de etnia Matis do DSEI Vale do Javari.

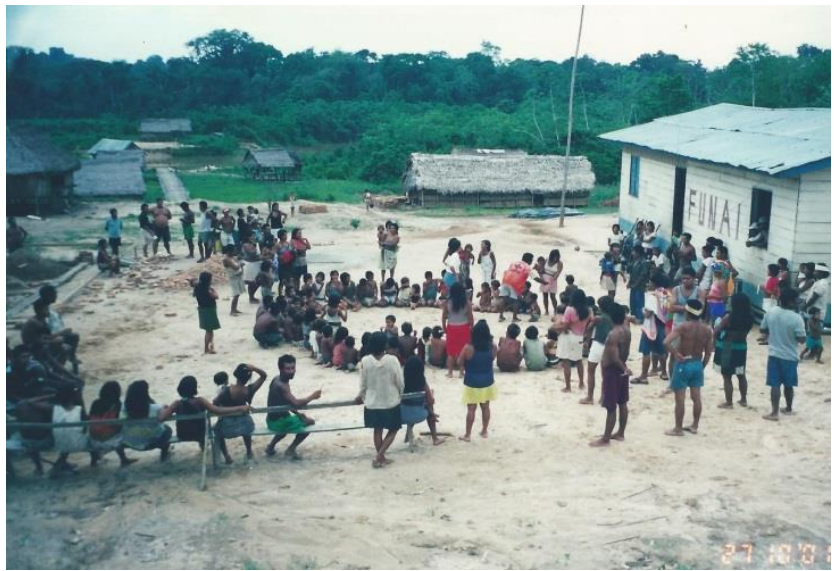

Figura 4: Ação coletiva na aldeia Massapê de etnia Kanamary do DSEI Vale do Javari.

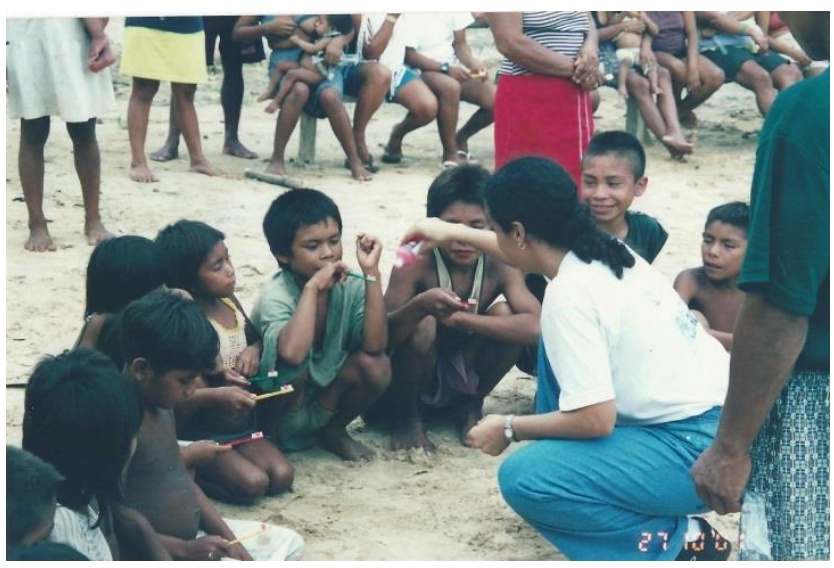

Figura 5: Ação coletiva assistida pelo AIS. Aldeia Massapê de etnia Kanamary do DSEI Vale do Javari.

- Capacitação dos agentes indígenas de saúde - AIS

A capacitação dos AIS aconteceram em serviço, no qual a equipe realizava reuniões com as lideranças e comunidade explicando como o trabalho seria desenvolvido, em seguida as palestras de promoção e prevenção de saúde bucal eram ministradas e o AIS realizavam a tradução para a comunidade presente. As Figuras 6 e 7 mostram a capacitação do agente indígena de saúde na aldeia São Sebastião de etnia Marubo. 


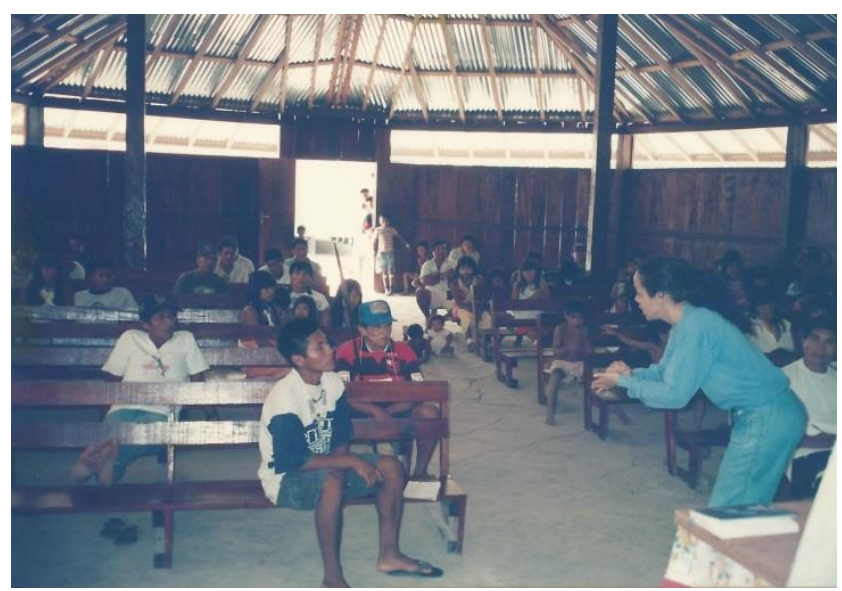

Figura 6: Palestra de promoção e prevenção de saúde bucal na aldeia São Sebastião de etnia Marubo.

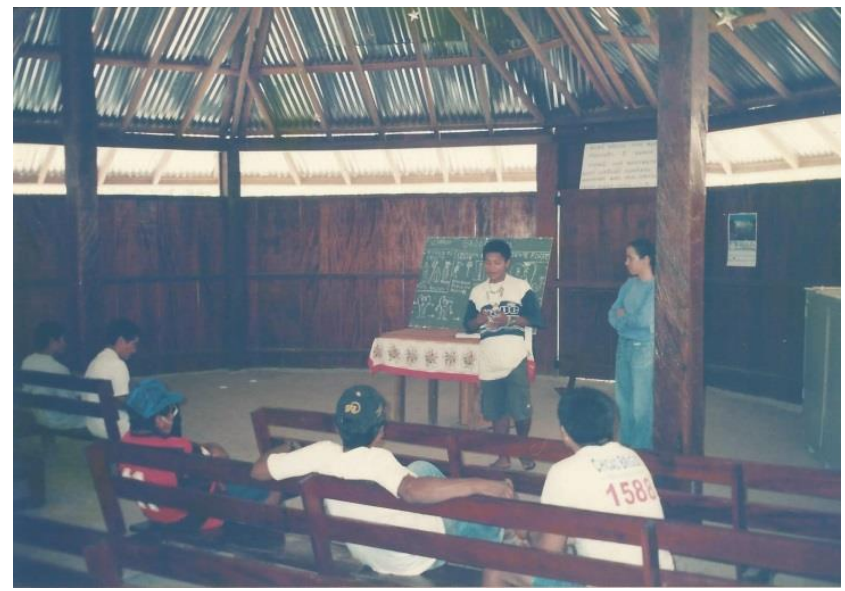

Figura 7: Capacitação do AIS na aldeia São Sebastião de etnia Marubo

\section{DISCUSSÃO}

Nos DSEI da Amazônia, por exemplo, são recorrentes as denúncias quanto à indisponibilidade de barcos ou aviões necessários para percorrer rapidamente as grandes distâncias entre as aldeias e as unidades de saúde. Em muitos casos, a falta de infraestrutura leva à morte. Como o caso de um idoso Mayuruna que, em fevereiro de 2013, veio a falecer na aldeia Fruta Pão porque o barco que servia ao DSEI de Atalaia do Norte/ AM estava sem combustível ${ }^{4}$.

Por outro lado, apesar dos territórios indígenas serem frequentemente impactados pela construção de empreendimentos para geração de energia, muitos polos-base sofrem com a falta de eletricidade. Mesmo quando há geradores, a instabilidade no fornecimento de combustível, ou a falta de manutenção adequada, pode levar ao frequente desligamento dos refrigeradores, afetando a continuidade de programas de vacinação ou levando ao desperdício de medicamentos ${ }^{4}$.

Outro risco importante à saúde indígena nesses contextos é a escassez de alimentos relacionada à diminuição ou degradação das áreas de plantio, da disponibilidade de pescado em rios cada vez mais poluídos por agrotóxicos e outros dejetos lançados nas bacias hidrográficas pelas áreas urbanas próximas ou ainda pela redução das áreas florestais pelo desmatamento. Isso explica um elevado número de casos onde foram relatadas situações de insegurança alimentar que são geradas ou intensificadas pela desapropriação territorial ou pela degradação ambiental $^{11}$.

Gomes salienta que "embora denominadas Casas de Saúde Indígena, essas estruturas não executam ações médico-assistenciais", pois, apesar de algumas vezes contarem com a presença de enfermeiros, técnico de enfermagem, auxiliares de enfermagem, assistentes sociais, psicólogos, médicos e farmacêuticos, estes estão presentes apenas para garantir os encaminhamentos necessários e o bemestar dos usuários indígenas durante sua estada.

Além disso, a contratação de profissionais de saúde para composição das equipes multidisciplinares de saúde indígena - EMSIs é outra demanda recorrente. Em pelo menos 11 casos, faltavam médicos e as equipes contavam apenas com enfermeiros, técnicos de enfermagem ou AISs para o atendimento. Mesmo quando os médicos estão presentes, eles visitavam as unidades esporadicamente, o que prejudicava o acompanhamento à saúde da população ${ }^{11}$.

Tanto as reivindicações indígenas quanto o programa federal podem ser entendidos como respostas a um problema fundacional do SASI (Subsistema de Atenção à Saúde Indígena). Desde os primeiros anos de atuação da Funasa como órgão responsável pelo SASI, a principal forma de operacionalização dos DSEIs é através da política de terceirização e do estabelecimento de convênios com repasses de recursos para organizações nãogovernamentais, prefeituras e associações indígenas para que estas executassem as ações de saúde ${ }^{11}$.

Na pesquisa de opinião realizada por Fontão ${ }^{2}$ et al 2017, os dados trazem elementos que se relacionam a outros atributos da atenção primária à saúde, em especial, a continuidade do cuidado e a construção de vínculo. Dos entrevistados que afirmaram a presença de médicos atendendo na comunidade antes do programa, 44,6\% responderam positivamente a pergunta sobre a busca de cuidados em saúde com especialistas indígenas. A mesma resposta positiva ocorreu em $41,1 \%$ daqueles que afirmaram que a comunidade teria ficado sem atendimento médico.

Segundo Guerra et $\mathrm{al}^{6}$ é de vital importância o treinamento de monitores indígenas para a execução de técnicas de higiene bucal, para que os mesmos sirvam de educadores em sua comunidade. Como não há especialistas indígenas na odontologia, verificou-se a necessidade de uma orientação mais especializada, por profissionais da área, para a realização de manobras higiênicas, fato que justifica a presença necessária e constante da equipe de saúde 
bucal, corroborando com os resultados do trabalho de Moimaz et $\mathrm{al}^{10}$.

Devem-se, ainda, avaliar as mudanças produzidas e seus significados nas dinâmicas sociais a partir da maior presença de médicos nas comunidades indígenas, considerando-se as assimetrias de poder estabelecidas na relação médicopaciente e na relação entre a biomedicina e outras formas de atenção à saúde ${ }^{2}$.

\section{CONCLUSÃO}

Diante de uma população com necessidades complexas e dificuldades logísticas, este estudo contribuiu com uma visão sobre a experiência dos cirurgiões-dentistas, que deve ser considerada parte interessada e importante na implementação e sustentabilidade do programa de saúde bucal para os povos indígenas.

\section{REFERÊNCIAS}

1. Aparício AB. Direitos territoriais indígenas: diálogo entre o direito e a antropologia - o caso da Terra Guarani "Morro dos Cavalos" [dissertação]. Florianópolis: Universidade Federal de Santa Catarina; 2008.

2. Fontão MAB, Pereira EL. More Doctors Project in indigenous health: reflections from an opinion survey. Interface (Botucatu). 2017;21(1):1169-80.

3. Fundação Nacional do Índio (Funai). Lista TI Brasil. Brasília: Funai; s.d. [acessado 2019 Jan 28]. Disponível em: http://goo.gl/22vqLf

4. Fundação Oswaldo Cruz (Fiocruz). Mapa de conflitos envolvendo injustiça ambiental e saúde no Brasil. 2015. Disponível em: http://goo. gl/7yEfm5. Acesso: 12 fevereiro 2019.

5. Gomes JS. "Casas de Saúde" e assistência farmacêutica: Desafios da saúde indígena em Belém e Macapá [dissertação]. Belém: Universidade Federal do Pará, Universidade Federal do Amazonas, Centro de Pesquisa Leônidas \& Maria Deane; 2008.

6. Guerra MAA. Treinamento de monitores indígenas em saúde bucal: uma experiência de trabalho com os Ticunas. Saúde em Debate. 1988; (n. esp.): $42-44$.

7. Instituto Socioambiental (ISA). Povos Indígenas no Brasil. Brasília: ISA; 2010. Disponível em: http://goo.gl/8xEJWe. Acesso: 27 janeiro 2019.

8. Instituto Brasileiro de Geografia e Estatística (IBGE). Os indígenas no Censo Demográfico 2010. Brasília: IBGE; 2012. [acessado 2019 Jan 28]. Disponível em: http://goo.gl/PyhXRf

9. Ministério da Saúde (BR). Fundação Nacional de Saúde. Política nacional de atenção à saúde dos povos indígenas. Brasília: Ministério da Saúde; 2002.

10.Moimaz SAS, Saliba NA, Garbin CAS, Bergamashi Júnior E, Souza JEA. Percepção de saúde bucal em uma comunidade indígena no Brasil. Rev Fac Odonto Lins. 2001; 13(1): 60-5.

11.Rocha DF, Porto MFS, Pacheco T. A luta dos povos indígenas por saúde em contextos de conflitos ambientais no Brasil (1999-2014). Ciênc saúde coletiva. 2019;24(2):383-92.

\section{CONFLITO DE INTERESSES}

Os autores declaram não haver conflitos de interesse.

\section{AUTOR PARA CORRESPONDENCIA}

\section{Tânia Adas Saliba}

tania.saliba@unesp.b
Submetido em 28/04/2019

Aceito em 15/05/2019 